\title{
HIV Accessory Proteins: Emerging Therapeutic Targets
}

\author{
Roger H. Miller and Nava Sarver \\ Targeted Interventions Branch, Basic Sciences Program, Division of \\ AIDS, National Institute of Allergy and Infectious Diseases, National \\ Institutes of Health, Bethesda, Maryland, U.S.A.
}

\section{HIV ACCESSORY PROTEINS}

In addition to the capsid (gag), polymerase (pol), and envelope (env) genes common to all retroviruses, human immunodeficiency virus type 1 (HIV-1) possesses six additional genes (i.e., nef, rev, tat, vif, vpr, and vpu) that encode regulatory proteins (Fig. 1). Four of these proteins, Nef, Vif, $\mathrm{Vpr}$, and $\mathrm{Vpu}$, were originally termed "accessory" proteins due to the fact that their function in vitro appeared to be nonessential for HIV replication (reviewed in Ref. 1). However, it is now evident that these proteins play an important role in viral replication in vivo, and some may be intimately involved in HIV pathogenesis and disease progression.

The following review is based on a recent symposium (HIV Accessory Proteins: Therapeutic Opportunities, February 2, 1995, Washington, DC) focusing on the therapeutic opportunities afforded by HIV accessory proteins.

\section{NEF}

Nef, a viral protein expressed early in infection, possesses two distinct functions: the ability to enhance HIV replication and the ability to downregulate the $\mathrm{T}$ cell surface $\mathrm{CD} 4$ molecule (reviewed in Ref. 2). Enhancement of virus replication results from the presence of virionassociated Nef that increases the infectivity of cell-free virus. This was revealed by studies comparing the replication capabilities of infectious provirus plasmids and virus particles in CEM

Address correspondence and reprint requests to: Nava Sarver, Targeted Interventions Branch, Basic Sciences Program, Division of AIDS, NIAID, NIH, Bethesda, MD 208927620. cells (a human $\mathrm{T}$ cell line) in a single-cycle assay where subsequent rounds of infection were inhibited by the addition of neutralizing antibody. Transfection of CEM cells with identical amounts of either a nef deletion $(\Delta)$ mutant or wild-type (wt) proviral DNA yielded similar expression levels of the HIV capsid protein p24. However, when CEM cells were infected with equal inocula of $\Delta n e f$ and wt virus (standardized for their p24 content), $\Delta$ nef virus yielded 3-to 5-fold less p24 than did the wt HIV-1. Thus, the presence of particle-associated Nef in wt virus resulted in an increase in p24 production in infected CEM cell.

The mechanism whereby Nef confers replicative advantage on the virus is not yet established. Comparison of virions with or without Nef revealed no difference in reverse transcriptase (RT) activity, viral binding, or viral entry into host cells. The reduction in infectivity of $\Delta$ nef virus does not seem to reflect on an inability to degrade CD4 (see below). When cells engineered to express a truncated CD4 molecule, lacking virtually the entire cytoplasmic domain required for Nef-induced CD4 degradation, were infected with $\Delta n e f$ or wt HIV, attenuation of $\Delta n e f$ virus growth was still observed.

The severe combined immunodeficient mouse implanted with human thymus and liver tissue (SCID-thy/liv) is an important animal model for studying HIV infection and pathogenesis. Mice are generated by surgical transplantation of human fetal liver and thymus fragments under the kidney capsule. After several months, the implanted tissues become fused together to form a graft, which is histologically and physiologically indistinguishable from a normal human thymus. Direct inoculation of the graft with HIV results in infection of human CD4-positive cells and precursor $\mathrm{CD} 4 / \mathrm{CD} 8$-double positive cells. 


\section{HIV-1 PROVIRAL GENOME}

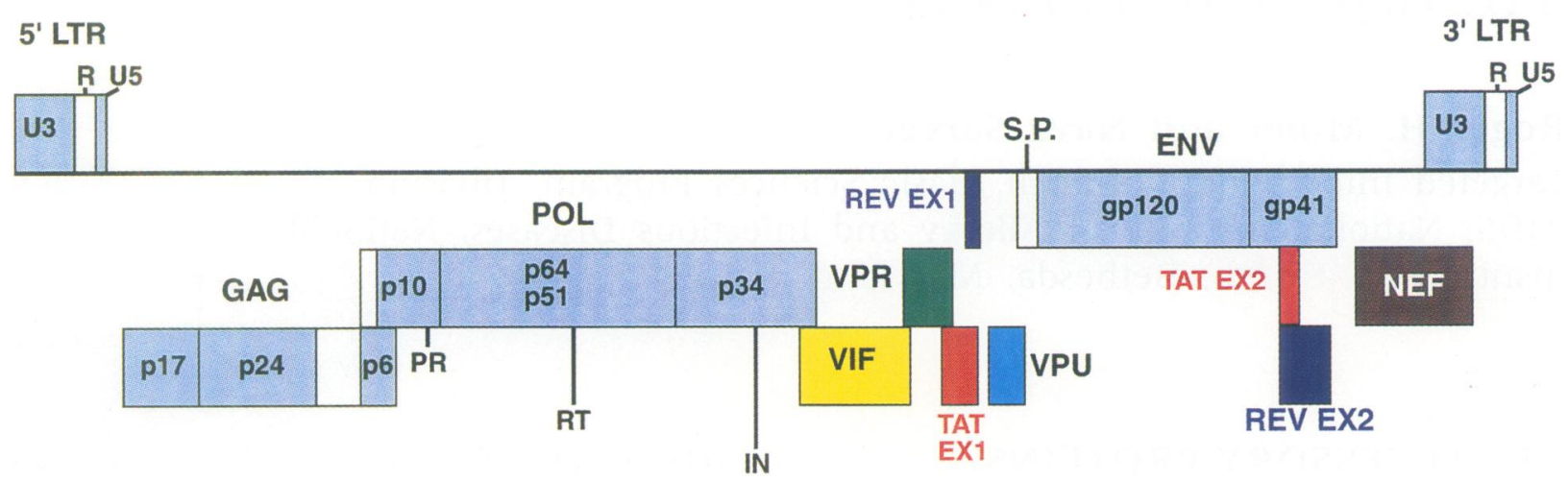

FIG. 1. Diagrammatic representation of the HIV-1 genome.

The long terminal repeats (LTRs), divided into the unique $3^{\prime}$ (U3), repeat (R), and unique 5' (U5) domains, flank the protein encoding regions of the virus genome (light blue). The large precursor proteins encoded by the capsid (GAG), polymerase (POL), and envelope (ENV) genes are proteolytically cleaved as depicted by the vertical lines. The GAG protein is cleaved into the smaller proteins p17, p24, and p6; the POL protein is cleaved into the protease (PR), reverse transcriptase (RT), and integrase (IN) proteins; and the ENV protein, with a signal peptide (S.P.) sequence at the amino terminus, is cleaved into the gp 120 (surface) and gp4l (transmembrane) proteins. Exons (EX) 1 and 2 of the rev and tat genes as well as the genes encoding the accessory proteins are highlighted by color.

The SCID-thy/liv model was used to evaluate the effect of HIV regulatory proteins on CD4-positive cells. CEM cells were transfected with infectious plasmids with deletions in the nef, vif, vpr, or vpu genes, and the virus produced was titered on activated human peripheral blood lymphocytes (PBLs). SCID-thy/liv mice were then inoculated with equal amounts of infectious virus. Such analysis revealed a hierarchy in the abilities of the four deletion mutant viruses to replicate and induce $\mathrm{CD} 4$ depletion in the graft relative to infection with wt HIV as follows: $\Delta v p r>\Delta v p u>$ $\Delta$ vif $>\Delta$ nef. Deleting the nef gene was thus more deleterious to virus replication than deleting any of the other regulatory genes studied.

Another well-established function of Nef is its ability to down-regulate CD4, a surface molecule crucial for helper $\mathrm{T}$ cell signaling and a principal cellular receptor for HIV. When nef gene constructs were expressed in CD4 positive cells, the half-life of CD4 was found to decrease from 24 to 4-6 hr. While expression of CD4 was unaffected, once the protein reached the cytoplasmic membrane Nef induced rapid endocytosis followed by lysosomal degradation of CD4 (3).
Recent work indicates that Nef may disrupt the interaction between CD4 and the p56 $6^{\text {lck }}$ tyrosine kinase (4). Site-directed mutagenesis of the cytoplasmic tail of CD4 (the binding site of p56 ${ }^{\text {lck }}$ and Nef) reveal that mutated CD4 molecules with decreased responsiveness to Nef also have reduced capacity to associate with $\mathrm{p} 56^{\mathrm{lck}}$. Amino acids 407 and 410 within a hydrophobic domain of CD4 are especially important. Thus, it is likely that Nef, or a Nef-recruited factor, competes with $\mathrm{p} 56^{\mathrm{lck}}$ for a binding site on the cytoplasmic tail of $\mathrm{CD} 4$ to disrupt $\mathrm{CD} 4 / \mathrm{p} 56^{\mathrm{lck}}$ interaction, thereby inducing CD4 endocytosis and its subsequent degradation.

\section{VPU}

Vpu is a regulatory protein encoded by HIV-1 but not by HIV-2 or most of the simian immunodeficiency viruses. This phosphorylated transmembrane protein has two distinct and separable functions in the HIV replication cycle: enhancement of viral export from infected cells and degradation of $\mathrm{CD} 4$ in the endoplasmic reticulum 
(ER). The requirement for Vpu in particle release is cell-type specific. Pulse-chase experiments utilizing constructs expressing HIV p6 Gag (a processed Gag peptide derived from the carboxylterminus of $\mathrm{p} 55 \mathrm{Gag}$ ) and/or Vpu indicate that the latter facilitates particle release in HeLa but not in COS cells. This finding implies the presence of a Vpu-interacting protein in some cells but not in others. Additional support for a cellular Vpu-interacting protein comes from a study showing that Vpu increases the release of heterologous retrovirus particles.

Using the yeast two-hybrid system a Vpu binding protein, or UBP, was identified. The protein, approximately $69 \mathrm{kD}$ in size, is encoded by a $2.9-\mathrm{kb}$ cellular transcript expressed in a variety of human tissues and cells, including PBLs. Partial analysis indicates that UBP shares certain sequence similarities with the immunophilin superfamily of proteins, which includes the cyclophilins and the FK506 binding proteins (FKBPs). It is still unclear what role UBP plays in mediating Vpu function(s) and whether UBP/Vpu interaction is required for HIV replication.

Insight into the mechanism of $\mathrm{Vpu}$-mediated viral export comes from solution NMR experiments demonstrating that the transmembrane domain of oligomeric Vpu has the potential to form an ion conductive membrane pore. Experiments in Xenopus oocytes verified the ion channel activity of synthetic Vpu. Interestingly, FKBP12, a member of the immunophilin family related to UBP, also interacts with a cellular protein involved in ion channel formation. While mutation of the transmembrane domain of $\mathrm{Vpu}$ resulted in the loss of Vpu-mediated ion channel formation activity and caused a reduction in virus release, there was no effect on $\mathrm{CD} 4$ degradation, indicating that these two functions of $\mathrm{Vpu}$ are separate.

The mechanism for Vpu-mediated CD4 degradation differs from that described for Nef. In HIV-infected cells, gp 160 Env and CD4 form complexes that become sequestered in the rough ER, preventing further transport of gp 160 and $\mathrm{CD} 4$ to the cellular membrane. Vpu reverses this inhibition of gp 160 sequestering by selectively degrading CD4 (5). Site-directed mutagenesis of Vpu suggests that both the anchor and cytoplasmic domains of the protein are required for this process.

As indicated, both the enhancement of viral secretion and the induction of CD4 degradation by $\mathrm{Vpu}$ involve distinct molecular mechanisms. It is not clear at this time whether blocking either function alone will have an impact on viral growth and pathogenesis or whether both functions will need to be targeted for maximal therapeutic benefit.

\section{VIF}

Several independent studies have shown that the Vif protein significantly increases the infectivity of HIV-1 particles. The importance of this protein is underscored by the observation that virtually all lentiviruses examined (the single exception being equine infectious anemia virus) possess a vif gene. While Vif was previously thought to be found only in the cytoplasm of infected cells (6), recent experiments have revealed the existence of Vif in mature virions. Studies with anti-Vif antibodies show that Vif exists within the virus core rather than on the virus surface, and it is estimated that 20 Vif molecules are encapsidated per virion.

Within the cytoplasm of infected cells, Vif exists both as soluble and as membrane-associated forms. Site-directed mutagenesis of vif revealed that the basic amino acids at the carboxyl terminus of the molecule (amino acids 157-160 and 173-184) are important for association with cell membranes. Interestingly, pretreatment of membranes with trypsin abolishes the ability of Vif to bind, suggesting that Vif interacts with a membrane-associated protein which stably anchors it to the membrane surface.

Again, these studies indicate an association between an HIV-1 regulatory protein, in this case Vif, with a cellular protein(s). Such proteins are likely to be required for the proper functioning of the regulatory proteins in viral replication and may provide additional targets for therapeutic intervention. One advantage in targeting a cellular "partner" over a viral gene product is that it obviates potential emergence of HIV escape mutants resistant to specific anti-viral agents.

In additional studies of the role of Vif in virus replication, Vif mutant virions purified from CEM cells (but not those purified from SupTl cells) were shown to exhibit a major defect in endogenous reverse transcriptase (RT) activity. Radiolabeled nucleotides were incorporated primarily into low molecular weight DNA fragments in the mutant virus. This suggests that a yet unidentified component of the virus core involved in reverse transcription is a target for Vif function. Although Vif does not effect RNA dimerization, the level of unprocessed p55 Gag 
in $\Delta$ vif virions was elevated compared with that in wt virions. At the present time, it is not clear what this effect on Gag processing means with regards to the role of Vif in HIV infectivity.

A second function of Vif involves reorganization of cytoskeletal elements, consisting largely of microtubules and intermediate filaments. While microtubules are composed of actin or tubulin, intermediate filaments are exclusively made of several proteins, notably vimentin. Experiments examining the cellular localization of Vif in HeLa cells transfected with an expression plasmid revealed that Vif associates specifically with intermediate filaments comprised of vimentin. Treatment of HeLa cells with brefeldin A, a drug that mediates the trafficking of membrane proteins from the ER to the Golgi, produced perinuclear caps of vimentin filaments. Vif expressed in HeLa cells colocalized specifically to such structures following drug treatment. While Vif does not affect the phosphorylation or solubility of vimentin, it causes a dramatic reorganization of the cytoskeleton. Vif may, therefore, also function to enhance the movement of the virion to the nucleus by reorganizing the cellular cytoskeletal elements. One hypothesis is that after the virion enters the host cell and uncoating begins, Vif attaches to vimentin and initiates the reorganization of intermediate filaments. Assisted by a vimentin-associated factor capable of moving along the filaments, the Vif-containing viral pre-integration complex is efficiently transported to the nucleus via a "three-dimensional" process, rather than a "two-dimensional" process in the absence of Vif. Conceivably, therapeutic agents that block Vif's ability to interact with vimentin are likely to interfere with the transport of the pre-integration complex to the nucleus, and dramatically affect the ability of HIV to efficiently infect lymphocytes.

\section{VPR}

Vpr exists as an oligomer (possibly a tetramer) in the nucleus of infected cells. Vpr is incorporated into virions via a specific mechanism involving p6 Gag (7). Virus strains encoding Vpr replicate faster and to higher levels than strains unable to encode a functional Vpr protein. Structural analysis of Vpr has focused on two fronts: determining the location of the nuclear localization signal (NLS) that facilitates transport of the nascent protein into the cell nucleus, and identifying the sequence(s) responsible for $\mathrm{Vpr}$ oligomerization (see below). Typical NLS elements consist of a stretch of positively charged amino acids. The NLS of Vpr is atypical in that it is located in a negatively charged domain at the amino terminus of the molecule. The Vpr NLS resides in a domain coincident with a region required for the interaction of the protein with a $180-\mathrm{kD}$ cellular factor that may facilitate transport of Vpr into the nucleus. Recombinant Vpr expressed in Escherichia coli is capable of forming oligomeric structures and preliminary work indicates that amino acids at the amino terminus of the molecule are required for oligomerization. Mutagenesis experiments mapped the crucial domain to amino acids 36-43. Speculations as to the purpose of $\mathrm{Vpr}$ oligomerization include expanding the capabilities of the small (i.e., $14 \mathrm{kD}$ ) monomeric protein and/or increasing the packaging efficiency of the protein.

The role of $\mathrm{Vpr}$ as an integral component of the virus may be explained by its profound biological effect on cells; Vpr was shown to regulate cell proliferation as well as cell differentiation $(8,9)$. As Vpr is released into the cell during uncoating of the virion, it is presumed to "prepare" an environment favorable to HIV replication and gene expression. In one study, an infectious molecular HIV clone was altered to express the murine Thy protein, a cell surface molecule that can serve as a convenient marker for monitoring virus gene expression. Using a dual staining method to analyze concomitantly the cell cycle status and the infection status of individual cells, infection with the altered HIV recombinant was shown to induce the accumulation of cells arrested in G2 in a variety of cell lines (PBL, SupT1, HeLa, and COS cells). In contrast, mock infected cells had twice as many cells in Gl than in G2. When the vpr gene was mutated, the recombinant infectious HIV could no longer induce $\mathrm{G} 2$ arrest. Interestingly, the vpr mutant virus was still capable of spreading and inducing syncytia. Another study examined the effects of baculovirus-expressed Vpr on tumor cell proliferation. While purified Vpr caused a dramatic inhibition of proliferation, latently infected cell lines treated with Vpr exhibited reactivation of virus replication. Such effects were the same whether recombinant Vpr or Vpr purified from HIV-infected sera were used. Overall, these studies implicate Vpr in inducing a long-lasting increase in cellular permissiveness to HIV replication by modulating the differentiation stage of the host cell.

These studies are consistent with recent find- 
ings demonstrating that viruses containing an intact $v p r$ gene are unable to establish a chronic infection of cultured lymphocytes due to the death of the infected cells (10). This effect is observed late in infection and occurs even in PBLs transfected with a provirus with a functional $v p r$ gene and a defective env gene. Since cell-to-cell spread does not occur under these circumstances, cell death must be mediated via Vpr synthesized de novo from the integrated provirus and not from import of virion-associated Vpr into the cell. Combined with other results, the data are consistent with the hypothesis that Vpr either prevents cells from continued proliferation or induces their terminal differentiation. The latter could explain why primary macrophages, which are differentiated cells, are capable of long-term expression of HIV without significant cell death. Additional experiments are necessary to determine the relevance of the in vitro observations to HIV replication in vivo.

How does Vpr exert such profound effects on cells? A recent report suggests that Vpr is linked to the glucocorticoid steroid pathway (11). A newly discovered cellular protein which associates with Vpr provides the link to this important regulatory pathway. Vpr-interacting protein 1 (Rip-1) is a $41-\mathrm{kD}$ cytosolic protein that is expressed in a wide variety of tissues and cells, including those that support HIV replication. Rip- 1 is translocated to the nucleus by both Vpr or glucocorticoid receptor (GR)-II-stimulating steroids. The finding that Vpr and Rip-1 coimmunoprecipitate with the human GR provides a potential biochemical mechanism for Vpr's activity and suggests the possible use of anti-glucocorticoid agents as inhibitors of HIV replication.

\section{PROSPECTS FOR THERAPY}

An emerging theme is that Nef, Vif, Vpr, and Vpu are involved in crucial aspects of HIV replication and release, cell susceptibility to infection, and modulation of signal transduction (Table 1). It is also apparent that several of these regulatory proteins share common functions mediated by different mechanisms, such as the downregulation of CD4 by both Nef and Vpu. While Nef promotes the internalization of surface CD4 and subsequent degradation in lysosomal vesicles, Vpu targets CD4 sequestered in the ER via a CD4-gp 160 complex. Why does the virus devote two gene products to CD4 down-regulation? It has been shown that removal of the HIV receptor from the cell surface benefits HIV replication by preventing superinfection and by enhancing the release of progeny virions; additional studies will determine whether CD4 down-regulation is important in other aspects of HIV infection of humans.

One of the most intriguing findings presented at the meeting was that the HIV regulatory proteins interact with specific cellular factors to perform their functions. Whatever the precise mechanisms involved, Nef, Vif, Vpr, and Vpu and their associated cellular proteins afford highly promising yet underexploited therapeutic opportunities. Conceivably, targeting cellular factors may be more effective than targeting viral factors. This premise is founded on the observation that cellular genes replicate with considerably more fidelity than HIV genes, thereby providing conserved targets for therapeutic interventions. Thus, the serious and prevalent problem associated with the evolution of virus variants resistant to the inhibitory effect of the drug(s) could be alleviated. Although a downside to targeting cellular factors is the potential cellular toxicity, it should be possible, nonetheless, to strike a clinical balance with significant antiviral effect and manageable toxicity. Importantly, it is possible that a synergistic inhibitory effect may be achieved in targeting the viral and cellular proteins simultaneously using combination therapies. Overall, efforts to exploit these dual targets should be explored in devising therapies against HIV infection which may be used alone or in combination with other anti-HIV therapeutic strategies.

Understanding the mode of action of the HIV regulatory proteins and their associated cellular factors opens a window for deciphering the intricate mechanism(s) associated with HIV-mediated cellular dysfunction and dysregulation. This knowledge, in turn, is imperative for developing effective therapies against HIV infection, either by identification of specific inhibitory agents in biochemical screening assays, a rational drug design approach driven by structure-function relationship, or by novel therapeutic strategies (e.g., gene-based inhibition).

\section{ACKNOWLEDGMENTS}

We thank the following investigators for their presentations at the meeting, some of which are summarized herein: Grace Aldronvandi, UCLA School of Medicine and Jonsson Comprehensive 
TABLE 1. HIV accessory proteins

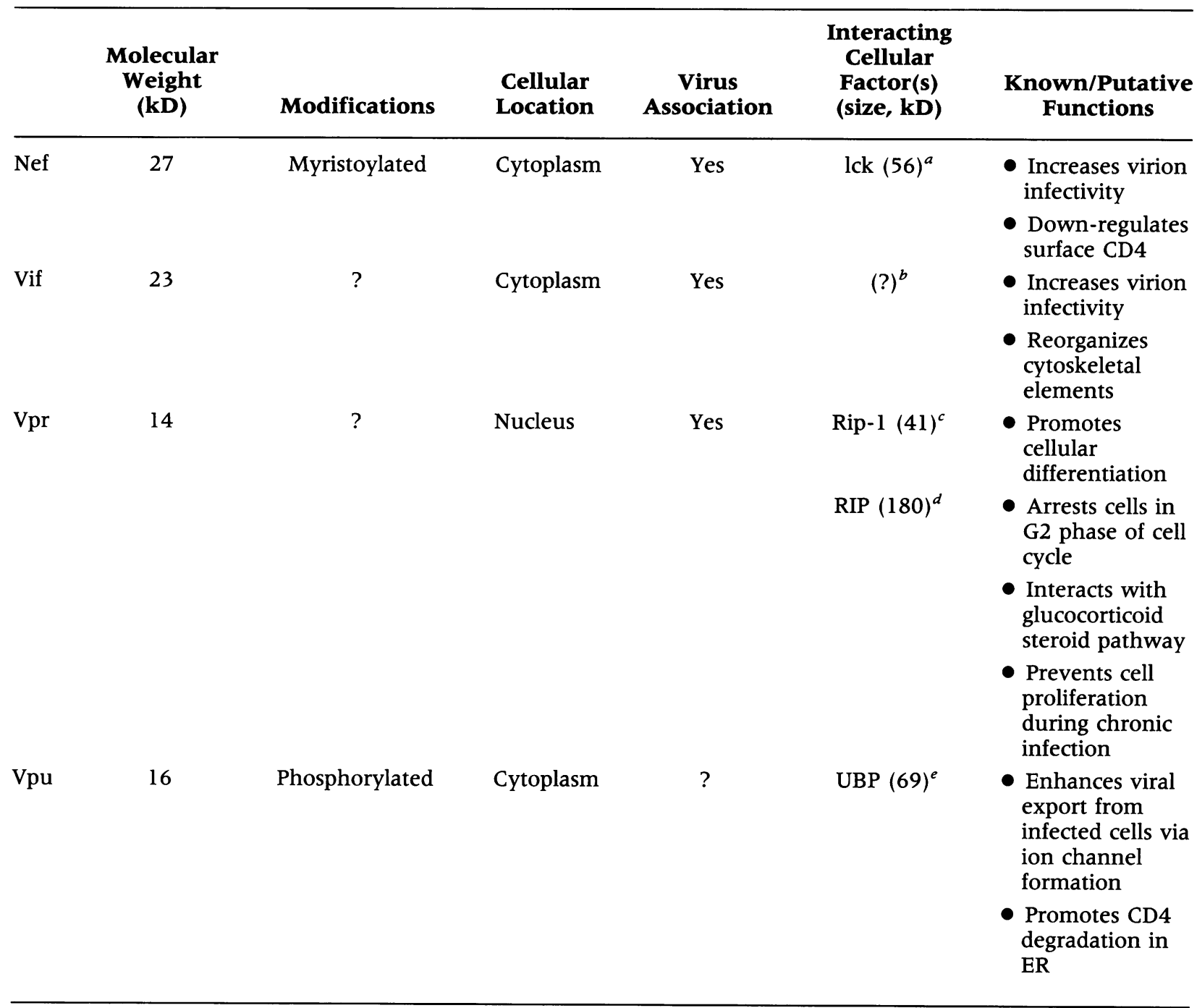

${ }^{a}$ Nef appears to compete for a CD4 binding site with the p56 ${ }^{\text {lck }}$ tyrosine kinase to induce endocytosis of CD4.

${ }^{b}$ Vif may interact with two uncharacterized cellular factors: (i) a membrane-associated protein which stably anchors Vif on the membrane surface and (ii) a vimentin-associated factor capable of moving a Vif-containing pre-integration complex along intermediate filaments.

'Rip-1 is a cytosolic protein that is translocated to the nucleus by Vpr. Rip-l coprecipitates with Vpr and the glucocorticoid receptor.

${ }^{d} \mathrm{RIP}$ is also reported to be involved in Vpr nuclear transport but the exact relationship of Rip-l and RIP has not yet been established. The fact that both Rip-1 and RIP appear to be involved in Vpr nuclear transport has led to the speculation that the latter represents a multimeric form of Rip-1.

${ }^{e}$ UBP shares similarities with the immunophilin family of proteins that includes the cyclophilins and the FK506 binding proteins. It has been speculated that UBP may be involved in the ion channel formation capability of Vpu.

Cancer Center; Dana Gabuzda, Dana Farber Cancer Institute; John Guatelli, UCSD and the San Diego Veterans Affairs Medical Center; M. Abdul Jabbar, The Cleveland Clinic Foundation; John Kappes, UAB; Nathaniel Landau, Aaron Diamond AIDS Research Center; Jon Marsh, National Institute of Mental Health; Antonio Pan- ganiban, University of Wisconsin; Vincente Planelles, UCLA School of Medicine and Jonsson Comprehensive Cancer Center; Ulrich Schubert, National Institute of Allergy and Infectious Diseases; Jacek Skowronski, Cold Spring Harbor Laboratories; Klaus Strebel, National Institute of Allergy and Infectious Diseases; Didier Trono, 
The Salk Institute for Biological Studies; Robert Vigne, INSERM; David Weiner, University of Pennsylvania; and Lingjun Zhao, University of Kansas Medical Center. We also thank Opendra Sharma for helpful suggestions on the program and Carl Dieffenbach for critical reading of the manuscript.

\section{REFERENCES}

1. Subbramanian RA, Cohen EA. (1994) Molecular biology of the human immunodeficiency virus accessory proteins. J. Virol. 68: 6831-6835.

2. Cullen BR. (1994) The role of Nef in the replication cycle of the human and simian immunodeficiency viruses. Virology 205: 1-6.

3. Aiken C, Konner J, Landau NR, Lenburg ME, Trono D. (1994) Nef induces CD4 endocytosis: Requirement for a critical dileucine motif in the membrane-proximal CD4 cytoplasmic domain. Cell 76: 853-864.

4. Salghetti S, Mariani R, Skowronski J. (1995) Human immunodeficiency virus type 1 Nef and $\mathrm{p} 56^{\mathrm{lck}}$ protein-tyrosine kinase interact with a common element in CD4 cytoplasmic tail. Proc. Natl. Acad. Sci. U.S.A. 92: 349-353.

5. Bour S, Schubert U, Strebel K. (1995) The human immunodeficiency virus type $1 \mathrm{Vpu}$ protein specifically binds to the cytoplasmic domain of CD4: Implications for the mechanism of degradation. J. Virol. 69: 1510-1520.

6. Goncalves J, Jallepalli P, Gabuzda DH. (1994) Subcellular localization of the Vif protein of human immunodeficiency virus type 1. J. Virol. 68: 704-712.

7. Paxton W, Connor RI, Landau NR. (1993) Incorporation of $\mathrm{Vpr}$ into human immunodeficiency virus type 1 virions: Requirement for the p6 region of gag and mutational analysis. J. Virol. 67: 7229-7237.

8. Levy DN, Refaeli Y, MacGregor BR, Weiner DB. (1994) Serum Vpr regulates productive infection and latency of human immunodeficiency virus type 1. Proc. Natl. Acad. Sci. U.S.A. 91: 10873-10877.

9. Levy DN, Refaeli Y, Weiner DB. (1995) Extracellular Vpr protein increases cellular permissiveness to human immunodeficiency virus replication and reactivates virus from latency. J. Virol. 69: 1243-1252.

10. Rogel ME, Wu LI, Emerman M. (1995) The human immunodeficiency virus type $1 \mathrm{vpr}$ gene prevents cell proliferation during chronic infection. J. Virol. 69: 882-888.

11. Refaeli Y, Levy DN, Weiner DB. (1995) The glucocorticoid receptor type II complex is a target of the HIV-1 vpr gene product. Proc. Natl. Acad. Sci. U.S.A. 92: 3621-3625. 